\title{
A critical note on the IAGA-endorsed Polar Cap index procedure: effects of solar wind sector structure and reverse polar convection
}

\author{
P. Stauning \\ Danish Meteorological Institute, Lyngbyvej 100, 2100 Copenhagen, Denmark \\ Correspondence to: P. Stauning (pst@dmi.dk)
}

Received: 12 February 2015 - Revised: 2 November 2015 - Accepted: 4 November 2015 - Published: 30 November 2015

\begin{abstract}
The International Association of Geomagnetism and Aeronomy (IAGA) has recently endorsed a new Polar Cap (PC) index version to supersede the previous seven different versions of the PCN (North) index and the five different PCS (South) index versions. However, the new PC index has some adverse features which should be known and taken into account by users of the index. It uses in its derivation procedure an "effective" quiet day level (QDC) composed of a "basic" QDC and an added solar wind sector term related to the azimuthal component $\left(B_{y}\right)$ of the interplanetary magnetic field (IMF). The added IMF $B_{y}$-related terms may introduce unjustified contributions to the PC index of more than 2 index units $\left(\mathrm{mV} \mathrm{m}^{-1}\right)$. Furthermore, cases of reverse convection during strong northward IMF $B_{z}$ (NBZ) conditions included in the database for calculation of index coefficients can cause unjustified index enhancements of $0.5-1 \mathrm{mV} \mathrm{m}^{-1}$ during calm conditions, reduction of index values by more than $20 \%$ during disturbed conditions, and inconsistencies between index coefficients and index values for the northern and southern polar caps. The aim here is to specify these adverse features and quantify their effects, and to suggest alternative steps for future modifications of the index procedure.
\end{abstract}

Keywords. Interplanetary physics (interplanetary magnetic fields) - magnetosphere physics (polar cap phenomena; solar wind-magnetosphere interactions)

\section{Introduction}

The Polar Cap (PC) index concept was suggested by Troshichev and Andrezen (1985) and developed roughly into its present form by Troshichev et al. (1988, 2006). The index has become an important parameter for solar-terrestrial relations and associated geomagnetic disturbances. PC index val- ues are primarily derived from the intensity of magnetic variations associated with the ionospheric forward two-cell convection patterns in the polar cap and scaled with respect to the driving interplanetary geoeffective electric field to make the index independent of local daily and seasonal variations. The PCN (North) index is based on geomagnetic variation data from Qaanaaq (Thule) in Greenland while the PCS (South) index is based on data from Vostok in Antarctica.

The PC indices have been used to derive interplanetary geoeffective electric fields (e.g. Troshichev et al., 2006), solar wind pressure pulses (Lukianova, 2003; Huang, 2005), cross polar cap voltage and polar cap diameter (Troshichev et al., 1996, 2000; Ridley and Kihn, 2004), ionospheric Joule heating (Chun et al., 1999, 2002), and general polar cap dynamics (Stauning et al., 2008; Fiori et al., 2009; Gao et al., 2012). The PC indices were also used to predict auroral electrojet intensities (Vennerstrøm et al., 1991; Vassiliadis et al., 1996; Takalo and Timonen, 1999), global auroral power (Liou et al., 2003), and ring current intensities (Stauning et al., 2008, Troshichev et al., 2011b, 2012). For specific space weather purposes the PC indices can be used to predict substorm development (Janzhura et al., 2007; Troshichev and Janzhura, 2009), and power line disturbances in the subauroral regions (Stauning, 2013c).

In the past there have been seven different versions of the PCN index and five versions of the PCS index (see Stauning, 2013b). The new PC index version endorsed by IAGA in 2013 and published at http://pc-index.org is supplied jointly by Arctic and Antarctic Research Institute (AARI), Russia, and DTU Space, Denmark. However, a comprehensive description of the PC index derivation procedure is not yet available. At the pc-index.org website reference is made to the note "Polar Cap (PC) Index" which, however, was written several years ago to 
describe a former PC index version developed at AARI. Thus, this description is not adequate now. In the documentation PC_index_description_main_document.pdf found at the Space DTU (2014) ftp site ftp: //ftp.space.dtu.dk/WDC/indices/pcn/PC_index_IAGA_ endorsement_documentation/, reference is made to the following sources for more extended descriptions of the PC index procedures: Troshichev et al. (2006); Janzhura and Troshichev (2008, 2011); Troshichev and Janzhura (2012). However, these references also describe previous PC index versions, which in some respects deviate substantially from the present IAGA-endorsed procedure. Further PC index documentation is found in the file PC_index_description_Appendix_A.pdf (hereinafter Appendix_AD), while a partial transcript of the MatLab program and some of the data files used to calculate PC index coefficients and index values are found in the directory: PC_index_description_AppendixA_file_archive (hereinafter Appendix_AF) at the above DTU Space ftp site.

The present note describes and quantifies adverse features in the IAGA-endorsed PC index procedure related to IMF $B_{y}$ effects on the QDC values used for the derivation of actual PC index values, and to the effects on the PC index scaling coefficients (slope and intercept) from including reverse convection events related to strong northward IMF $B_{z}$ (NBZ) conditions in the database used for the parameter derivation. The goal is to document the effects of these problems in order to make them known to possible users of the IAGA-endorsed PC index values. Alternative steps in the index procedure are suggested for future modifications of the IAGA-endorsed procedure.

\section{Polar convection modes and PC index basics}

\subsection{Convection modes}

Polar magnetic variations beyond the quiet daily variations (QDC) are predominantly caused by the horizontal and field-aligned currents related to the convection systems sketched in Fig. 1. The horizontal currents are equivalent to oppositely directed ionospheric drift motions. The DP2 (forward) and DP3 (reverse) convection modes could be considered the basic modes for the transpolar convection and currents while DP1 (substorm) and DP4 (DPY) convection modes may generate perturbations of the two basic transpolar convection systems.

\subsection{PC index definition}

The basic definition of the Polar Cap (PC) index could be found in Troshichev et al. (2006). In summary (cf. Stauning, 2013b), the PC index is based on an assumed linear relation between $E_{\mathrm{M}}$, the "geo-effective" (or "merging") electric field in the solar wind encountering the Earth, and $\Delta F_{\mathrm{PROJ}}$, the
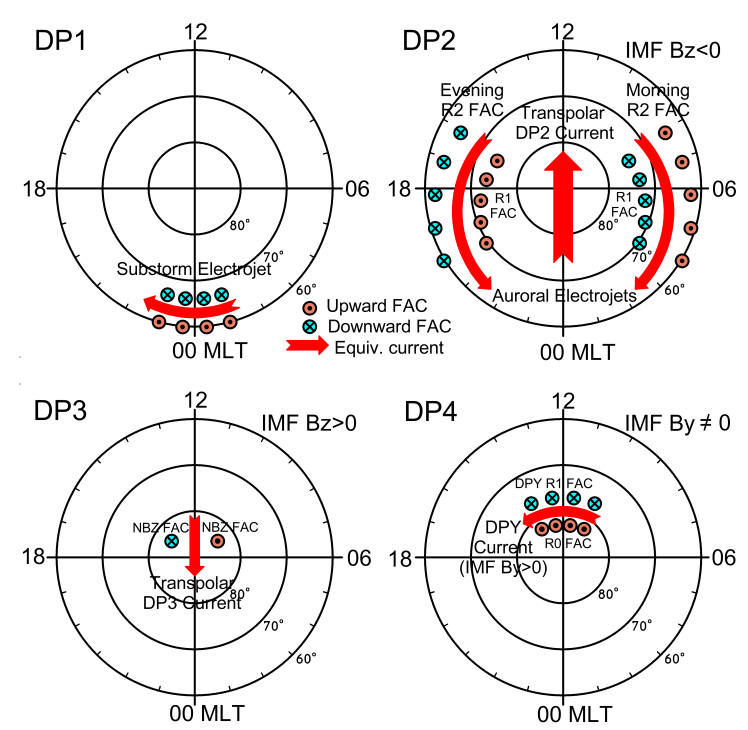

Figure 1. Sketches of ionospheric and field-aligned currents related to DP1 (substorm), DP2 (forward), DP3 (reverse), and DP4 (DPY) polar convection systems.

polar cap horizontal magnetic variation (at ground) projected to the so-called "optimum direction".

The linear relation is

$\Delta F_{\mathrm{PROJ}}=\alpha E_{\mathrm{M}}+\beta$.

The optimum direction is perpendicular to the average DP2 transpolar equivalent current direction (see Fig. 1) and makes an angle $\varphi$ to the dawn-dusk direction. The projection enhances the coupling of the PC index to the dominant DP2 forward convection mode.

The "geo-effective" (or "merging") electric field (Kan and Lee, 1979) is defined through

$E_{\mathrm{M}}=V_{\mathrm{SW}} B_{\mathrm{T}} \sin ^{2}(\theta / 2)$,

where $V_{\mathrm{SW}}$ is the solar wind velocity, $B_{\mathrm{T}}$ is the transverse component of the interplanetary magnetic field $\left(B_{\mathrm{T}}=\right.$ $\sqrt{\left(B_{y}^{2}+B_{z}^{2}\right)}$, while $\theta$ is the polar angle between the $Z$ axis of the geocentric solar-magnetospheric (GSM) coordinate system and the transverse IMF component.

Equation (1) is now inverted to give a definition of the PC index by equivalence with the merging electric field measured in $\mathrm{mV} \mathrm{m}^{-1}$ :

$\mathrm{PC}=\left(\Delta F_{\mathrm{PROJ}}-\beta\right) / \alpha\left(\sim E_{\mathrm{M}}\right)$.

The scaling parameters comprise the optimum direction angle, $\varphi$, derived to give optimum correlation between the solar wind intensities and the projected magnetic variations, while the coefficients, $\alpha$ (slope) and $\beta$ (intercept), are found from Eq. (1) through regression analyses based on an ensemble of concurrent values of the merging electric field, $E_{\mathrm{M}}$, and the polar cap horizontal magnetic variation vector, $\Delta \boldsymbol{F}$, counted from the quiet level, $\boldsymbol{F}_{\mathrm{QL}}$. 


\subsection{Initial QDC procedure}

The initial concept of the reference quiet day curve (QDC) for Polar Cap (PC) index calculations was defined in Troshichev et al. (2006) (hereinafter TJS2006) by the sentence: "Magnetic deviations $\delta D$ and $\delta H$ are calculated from a certain level, 'curve of quiet day', which presents the daily magnetic variation, observed at the particular station during extremely quiescent days."

The initial QDC procedure described in Janzhura and Troshichev (2008) (hereinafter JT2008) is based on the above principle. Each element of the two components of an initial QDC value is derived from quiet data values recorded at the same time of day within an interval of 30 days. The date for the calculated QDC is found as the weighted average of all dates with quiet segments. Successive displacements of the 30-day interval provide a series of QDC data sets from which non-linear interpolation provides QDC values for each day. The selection relies on quiescence criteria based on limits regarding the variability and the gradient of the respective component data within $20 \mathrm{~min}$ intervals. The concept from TJS2006 is violated in the new index procedure by the addition of an IMF $B_{y}$-related solar wind sector (SS) term derived from the daily median of all data, quiet as well as disturbed, to the basic QDC derived from quiet data samples only.

In the initial (JT2008) QDC procedure the separations within the 30-day interval between the dates of the quiet samples and the actual QDC date are not considered. Thus, the possible modulation of the basic QDC values with the phase of the solar 27-day rotation cycle, in particular with the solar wind sector-related phase of systematic variations in the IMF $B_{y}$ component, is lost. This is seen clearly in Fig. 4 of JT2008 where the displayed variations in QDC amplitudes through November months during all years 1998-2002 are almost perfectly linear to indicate slow seasonal changes only.

\section{IMF $B_{y}$-related solar wind sector effects in the IAGA-endorsed QDC procedure}

\subsection{Derivation of the IMF $B_{y}$-related solar wind sector term}

The IAGA-endorsed QDC procedure, which includes a solar wind sector (SS) contribution, is described briefly in the notes found in the PC index documentation (Appendix_AD).

The "Note on calculation 2 (sector structure)" reads:

"The sector structure is determined for each minute by a two-step smoothing process from the THL (Thule) $X$ and $Y$ component daily median values, respectively. In the first step, a 7-day running mean is produced, which in the next step is again smoothed by a 7-day "Robust Loess" (quadratic fit)."

The solar wind sector (SS) contributions, $\Delta H_{\mathrm{SS}}$, determined from the daily median values of the $H$-components, are presented in Fig. 6a, b of Janzhura and Troshichev, 2011 (hereinafter JT2011) for June 1996 and 2001. Examples of smoothing and processing over various intervals are shown in the figures. The variations in the final $\Delta H_{\mathrm{SS}}$ term through any single day are generally small (here less than $\pm 5 \mathrm{nT}$ ) compared to the amplitude of the total variation $(-35$ to $+65 \mathrm{nT}$ ) through June 2001.

A similar presentation could be made for the geomagnetic $D$-component. Examples of the solar wind sector terms for the $D$-component are provided in JT2011. Note, however, that there are inconsistencies between the scaling of the SS effects in Figs. 3, 6, and 7 vs. Figs. 4 and 8 in JT2011 to be discussed in Sect. 5.2 below.

\subsection{Solar wind sector effects on the "basic" QDC derivation}

A quite new feature in the IAGA-endorsed QDC derivation procedure is the subtraction of the SS terms from the measured component data values thereby changing the data base used to derive the "basic" QDC. This feature is included neither in JT2011 nor in the "Polar Cap (PC) index" documentation available at http://pc-index.org, but found only by analysing the computer procedure "qday_db.m" in Appendix_AF that reads

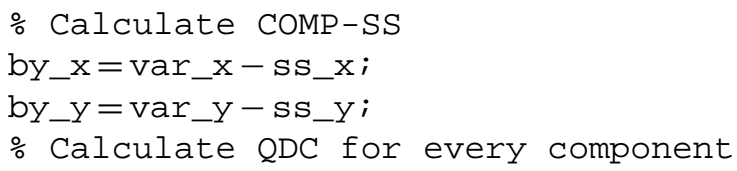

The effects of this modification of the data basis for QDC calculations depend on the relative position of quiet intervals with respect to the IMF $B_{y}$ phase. They are possibly small but uncertain, which stresses (again) the need for a comprehensive and updated description of the IAGAendorsed PC index procedure. Moreover, the files holding the solar sector terms (ss_x, ss_y) and the derived QDC values (qdc_x, qdc_y) are not available in Appendix_AF for independent examination.

\subsection{The "effective" QDC in the IAGA-endorsed PC index procedure}

The IAGA-endorsed "effective" QDC is defined in "Note on calculation 5 (geomagnetic disturbances in observatory data)" found in Appendix_AD: "Subtract sector structure and QDC from THL $X$ and THL $Y$ and make 5 min averages". The corresponding computer procedure "dist $5 \mathrm{~m} . \mathrm{m}$ " found in Appendix_AF reads

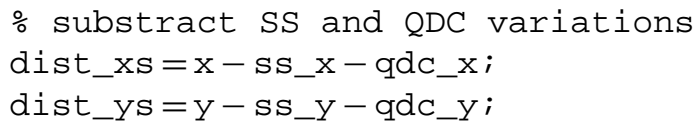

Thus, an "effective" quiet day level is composed of a "basic" QDC (TJS2006; JT2008) with an added solar 


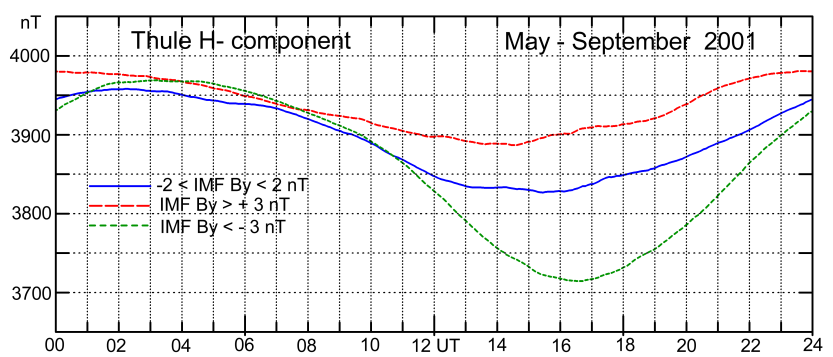

Figure 2. Mean daily variation in the $H$-component at Thule during summer 2001 derived for three gradations of the IMF azimuthal component: $B_{y}>3 \mathrm{nT}$ (red line), $-2<B_{y}<2 \mathrm{nT}$ (blue line), and $B_{y}<-3 \mathrm{nT}$ (green line) (cf. Fig. 5b of JT2011).

wind sector (SS) contribution as explained in JT2011. It is important to note that all elements of the basic QDC are derived from quiet samples recorded at corresponding times of the day while the added SS term is based on daily median values of all data whether quiet or disturbed. The main problem for the "effective" QDC now defined in the IAGA-endorsed procedure relates to the solar wind sector contributions as pointed out in the critical commentary by Stauning (2013a).

Here, we resolve the horizontal geomagnetic terms in orthogonal $(H, D)$ components in order to use the examples of the IMF $B_{y}$-related vector, $\boldsymbol{\Delta} \boldsymbol{F}_{\mathrm{SS}}$, provided in JT2011. The "effective" QDC values for the geomagnetic $H$-component are produced by adding IMF $B_{y}$-related terms, $\Delta H_{\mathrm{SS}}$ (presented for June 2001 in Fig. 6b of JT2011) to the initial basic QDCs determined (approximately) by the method described in JT2008. The result is presented in Fig. 1 of JT2011 for days 145 through 245 of the summer of 2001 with the QDC curve in black superimposed on the magnetic variation data in faint grey or in Fig. 4.10 (in colour, more readable) of Troshichev and Janzhura (2012) (hereinafter TJ2012).

When adding the $\Delta H_{\mathrm{SS}}$ terms to the smoothly varying basic QDC- $H$ values, the $\Delta H_{\mathrm{SS}}$ variation patterns are transferred to become modulations of the "effective" QDC- $H$ component patterns as seen most easily in the upper envelope corresponding to night values, and in the lower envelope for midday values of the QDC- $H$ curves shown in Fig. 1 of JT2011 (or in Fig. 4.10 of TJ2012) The QDC- $D$ component including a $\Delta D_{\mathrm{SS}}$ term is handled correspondingly.

Note from these figures that the upper and lower envelopes of the "effective" QDC- $H$ traces are almost congruent such that the in-between daily range of the "effective" QDC- $H$ series only shows slow seasonal variations with maximum amplitude at midsummer. When $\Delta H_{\mathrm{SS}}$ varies monotonically (with constant slope), the modulation patterns of the upper and lower envelopes are identical apart from half a day's phase shift. When the $\Delta H_{\mathrm{SS}}$ slope changes, then small differences between the upper and lower envelopes may result. The display in Fig. 1 of JT2011 (Fig. 4.10 of TJ2012) presents these characteristics.
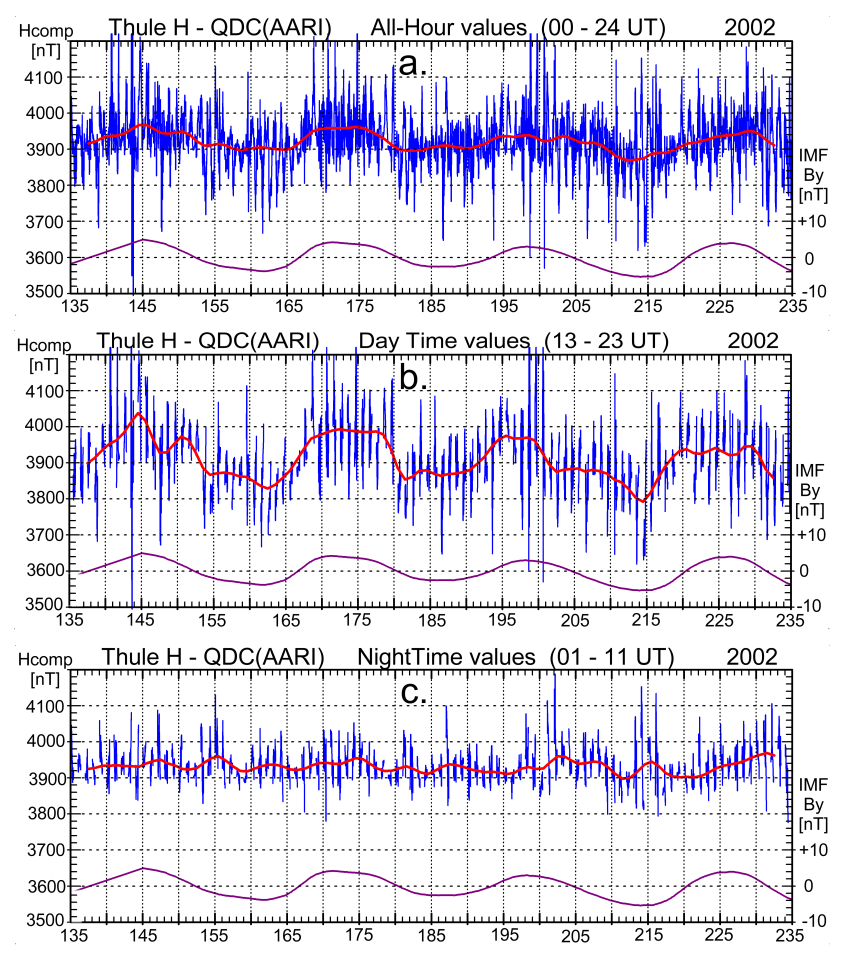

Figure 3. Data 2002. Thule $H$-comp - QDC(AARI) in blue line for: (a) all hours, (b) daytime, (c) night-time hours. Smoothed median values in red line. IMF $B_{y}$ in magenta line (scale to the right).

There are two improper features in the "effective" QDCs presented in these figures:

A. The top of the QDC- $H$ traces present calculated night values of the quiet magnetic field, but the real night values are not significantly affected by the IMF $B_{y^{-}}$ variations (cf. Figs. 2 and $3 \mathrm{c}$ here). Hence, the upper envelope of the "effective" QDC should be rather flat and not strongly modulated by IMF $B_{y}$ variations.

B. The amplitude ranges between the upper and lower envelopes of the presented QDC- $H$ traces are not affected by the IMF $B_{y}$ variations, but the real daily variation ranges in the magnetic field are significantly different for positive and negative IMF $B_{y}$ cases (see Fig. 2).

\section{IMF $B_{y}$ modulation of the horizontal polar geomagnetic field components}

\subsection{Statistics for IMF $B_{y}$ modulation of polar magnetic fields}

The addition of the solar wind sector term to the basic QDC in the new IAGA-endorsed PC-procedure is not justified since the day and night changes in the magnetic components with IMF $B_{y}$ are quite different and, therefore, could not be compensated for by just adding a slowly varying 
daily term. Statistically, the difference between IMF $B_{y}$ effects at day and night is easily seen in Fig. 2 (cf. Fig. 5 of JT2011), which displays the mean daily variation in the $H$ component recorded at Thule during the summer months for three gradations of the IMF $B_{y}: B_{y}<-3 \mathrm{nT},-2<B_{y}<2 \mathrm{nT}$, and $B_{y}>3 \mathrm{nT}$. With magnetic local time (MLT) noon located at 15:00 UT and local time (LT) noon at 16:00 UT it is easy to see that there is hardly any IMF $B_{y}$-related difference between the three gradations through local night $(\sim 00: 00-12: 00$ UT) while there are substantial differences at local daytime $(\sim 12: 00-24: 00 \mathrm{UT})$.

Note also from Fig. 2 that the amplitude range in the mean daily variation varies with the IMF $B_{y}$ parameter. For summer 2001, from midnight ( 04:00 UT) to noon $(\sim 16: 00$ UT) the amplitudes in the daily variation for the three cases are $\sim 70,120$ and $230 \mathrm{nT}$ for positive, near-zero, and negative IMF $B_{y}$ gradations, respectively. Corresponding large variations should appear in the daily amplitudes of the "effective" QDC- $H$ component displayed for the same period in Fig. 1 of JT2011 (or Fig. 4.10 of TJ2012) but are not seen.

The display in Fig. 2 relates to all conditions and not just the quiet cases, but the trends are the same for just the quiet cases. Thus, imposing on the basic QDC- $H$ series the (almost) same $\Delta H_{\mathrm{SS}}$ shift at night and at day is not in agreement with the statistics presented in Fig. 2. It could be noted from Fig. 1 of JT2011 (or Fig. 4.10 of TJ2012) that the amplitude of the variations in both the upper and lower envelope of the "effective" QDCs through June (days 152-181) are $\sim 100 \mathrm{nT}$, which corresponds to the range in the $\Delta H_{\mathrm{SS}}$ variation $(-35$ to $+65 \mathrm{nT})$ through June 2001 shown by the magenta line in Fig. 6b of JT2011.

These objections were published in Ann. Geophys. in a commentary, Stauning (2013a), to the paper JT2011. The commentary paper was submitted in 2012 and forwarded at that time to the authors of JT2011, but no reply has been published yet.

\subsection{IMF $B_{y}$ modulations of Thule magnetic data at daytime and at night-time}

Magnetic data from Thule have been examined for a closer inspection of the different day and night response in the $H$ and $D$-components to IMF $B_{y}$ variations. Plots were made to present summer data from mid-May to mid-August (days 135 to 235). For each year the component data have been divided into all-hour (00:00-24:00 UT), daytime (13:00-23:00 UT), and night-time (01:00-11:00 UT) groups. The $H$-component data from these groups are displayed in separate plots in the example for 2002 in Fig. 3.

In order to reduce the ordinary daily variations, the regular quiet day variations have been suppressed by subtracting the basic QDC values from the recorded data using QDC files (without the solar wind sector contributions) calculated by the JT2008 procedure and supplied from AARI. Further- more, from the displayed $H$-components the daily median values have been derived. With a weighted smoothing over 5 days these median values are shown in heavy red line. For comparison, the related IMF $B_{y}$ values (smoothed over 7 days) are presented in magenta line in the lower part of the diagrams with their scale values indicated to the right.

From Fig. 3 it is seen that the IMF $B_{y}$ variations displayed by the magenta line in the bottom of each field are reproduced to some extent in the all-hour plot of the $H$-component in Fig. 3a. However, it is clear that the strong IMF $B_{y}$-related variations are only reflected in the daytime values displayed in Fig. 3b. For the night-time values of the $H$-component displayed in Fig. $3 \mathrm{c}$ there is no correspondence between the variations in the $H$-component and the IMF $B_{y}$ changes. Corresponding results appear in similar diagrams for further years (1997-2009) looked at.

\section{Effects of the IAGA-endorsed "effective" QDC procedure on PC index values}

\subsection{Calculation of the effects of the solar wind sector term}

To derive PC index values, the magnetic variation vector, $\boldsymbol{\Delta} \boldsymbol{F}$, corrected for the "effective" QDC (QDCeff) should be projected to the optimum direction characterized by the angle, $\varphi$. Then the intercept value, $\beta$, should be subtracted, and the result should be divided by the slope, $\alpha$. Thus, for the magnetic variation vector:

$$
\boldsymbol{\Delta} \boldsymbol{F}=\boldsymbol{F}_{\mathrm{RAW}}-\boldsymbol{F}_{\mathrm{QDCeff}}=\boldsymbol{F}_{\mathrm{RAW}}-\boldsymbol{F}_{\mathrm{QDC}}-\boldsymbol{\Delta} \boldsymbol{F}_{\mathrm{SS}} .
$$

Here, for Thule $(77.47 \mathrm{~N}, 290.77 \mathrm{E}$ ), with declination (year $2001)=297.33^{\circ}$, the optimum angle and slope values presented at http://pc-index.org have been used in combination with $\Delta \boldsymbol{F}_{\mathrm{SS}}$ vectors comprising the components $\Delta H_{\mathrm{SS}}$ and $\Delta D_{\mathrm{SS}}$ presented in JT2011 (SS $\left(H_{\mathrm{THL}}\right)$ and $\left.\mathrm{SS}\left(D_{\mathrm{THL}}\right)\right)$. For the projection of the SS contributions to the optimum direction we have used

$$
\Delta F_{\mathrm{SS}, \mathrm{PROJ}}=\Delta H_{\mathrm{SS}} \sin \left(V_{\mathrm{PROJ}}\right)-\Delta D_{\mathrm{SS}} \cos \left(V_{\mathrm{PROJ}}\right),
$$

where

$V_{\mathrm{PROJ}}=$ longitude - declination $+\mathrm{UThr} \times 15^{\circ}+\varphi$.

Using the relation in Eq. (3) the SS-related contribution to the PC index is then

$\Delta \mathrm{PCN}_{\mathrm{SS}}=-\Delta F_{\mathrm{SS}, \mathrm{PROJ}} / \alpha$.

The optimal direction at noon is close to perpendicular to the IMF $B_{y}$-related contributions. Hence the projection of the SS vector, $\boldsymbol{\Delta} \boldsymbol{F}_{\mathrm{SS}}=\left(\Delta H_{\mathrm{SS}}, \Delta D_{\mathrm{SS}}\right)$, to the optimum direction is small at noon. At other local hours the projection angle changes and the projected values of the (almost constant) SS 
Table 1. Sector-related contributions to PCN index on 22 June 2001 according to index parameters from http://pc-index.org and $\boldsymbol{\Delta} \boldsymbol{F}_{\mathrm{SS}}$ values from JT2011.

\begin{tabular}{lllrrr}
\hline Hour $(\mathrm{UT})$ & Opt angle $(\varphi)$ & Slope $(\alpha)$ & $V_{\text {proj }}$ & $\Delta F_{\mathrm{SS}, \text { proj }}$ & $\Delta \mathrm{PCN}_{\mathrm{SS}}$ \\
\hline 00.00 & $55.22^{\circ}$ & 40.21 & $48.66^{\circ}$ & $22.38 \mathrm{nT}$ & -0.56 \\
06.00 & $43.44^{\circ}$ & 31.61 & $126.88^{\circ}$ & $76.00 \mathrm{nT}$ & -2.40 \\
12.00 & $18.46^{\circ}$ & 45.22 & $191.90^{\circ}$ & $25.74 \mathrm{nT}$ & -0.57 \\
15.00 (noon) & $18.09^{\circ}$ & 65.17 & $236.53^{\circ}$ & $-32.16 \mathrm{nT}$ & +0.49 \\
18.00 & $40.48^{\circ}$ & 54.54 & $303.92^{\circ}$ & $-76.26 \mathrm{nT}$ & +1.40 \\
\hline
\end{tabular}

vector become more significant in magnitude and shift sign twice. The diminished slope values, $\alpha$, away from midday cause further enhancement (cf. Eq. 7) of the effects from the SS contribution to the PC index values during local night and morning hours.

\subsection{Example case for 22 June 2001}

The next question is now: what are the consequences of using the "effective" QDC procedure (Appendix_AF) for the PC index calculations? Here, we use the data published by the authors of the procedure to derive the additions to the PCN index values from the solar wind sector terms. The solar wind sector effects are mainly associated with IMF $B_{y}$ related currents (DP4 convection mode, cf. Fig. 1) in and around the daytime Cusp region at latitudes equatorward of Thule, the current direction being related to the sign of $B_{y}$. This current system is located at and close to local noon and its effects on magnetic recordings from Thule are very clear for the daytime sector in the data plots in Fig. 3b as well as in the statistical daytime values presented in Fig. 2. It should be noted that since the solar wind sector-related disturbance vectors, $\boldsymbol{\Delta} \boldsymbol{F}_{\mathrm{SS}}$, are derived from smoothed daily medians, they keep an almost constant value and direction in the local (rotating) reference frame through day and night and from one day to the next.

Since we need both the $\Delta H_{\mathrm{SS}}$ and the $\Delta D_{\mathrm{SS}}$ terms to calculate $\triangle \mathrm{PCN}_{\mathrm{SS}}$, example values could have been extracted from Fig. 4b in JT2011, where the peak values seen on 22 June 2001 are also the peak values in the displays in their Fig. 8. There is a problem, however. The example values, counted from the levels at IMF $B_{y}=0$, would then be $\Delta H_{\mathrm{SS}}=150 \mathrm{nT}, \Delta D_{\mathrm{SS}}=90 \mathrm{nT}$. Control calculations of the 5-day sliding averages of the median $\Delta H_{\mathrm{SS}}$ and $\Delta D_{\mathrm{SS}}$ terms for June 2001 agree with the concept that the values in Fig. $6 \mathrm{~b}$ of JT2011 are correct while the values presented in their Figs. 4 (with the scale factor 10) and in Fig. 8 are around twice the correct values. Hence, for calculations of the IMF $B_{y}$ modulation of the PCN index we use here:

$\Delta H_{\mathrm{SS}}=65 \mathrm{nT} ; \Delta D_{\mathrm{SS}}=40 \mathrm{nT}$

(on 22 June 2001 at IMF $B_{y} \sim 4 \mathrm{nT}$ ).

Using these values for the present case from 22 June 2001 where IMF $B_{y} \sim 4 \mathrm{nT}$, the solar wind sector (SS) related

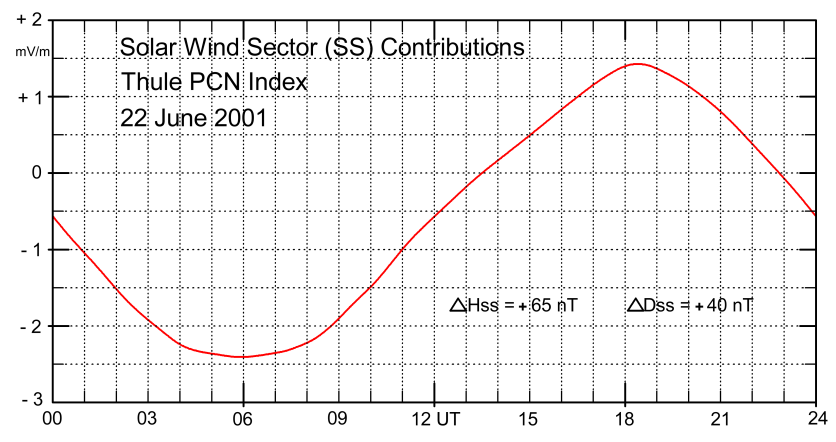

Figure 4. IMF $B_{y}$-related solar wind sector (SS) contributions to the PCN index values derived according to the "effective QDC" procedure defined in JT2011 with the excursions $\Delta H_{\mathrm{SS}}=65 \mathrm{nT}$ defined in their Fig. $6 \mathrm{~b}$ and $\Delta D_{\mathrm{SS}}=40 \mathrm{nT}$. Index coefficients are from http://pc-index.org.

changes, $\triangle \mathrm{PCN}_{\mathrm{SS}}$, in the index values have been calculated and are displayed in Fig. 4 . The contributions, $\triangle \mathrm{PCN}_{\mathrm{SS}}$, related to the $\Delta H_{\mathrm{SS}}$ and the $\Delta D_{\mathrm{SS}}$ terms, which change little during the day or from day to day (see Fig. 6b of JT2011), come on top of the PCN values calculated from the actual magnetic variations corrected for the basic QDC.

Table 1 displays the SS contributions to the PCN index from the solar wind sector terms given in Eq. (8) for some selected times through the day. Like for Fig. 4, the coefficients, optimum angle and slope values are taken from the IAGA-endorsed files of coefficients provided at the web site http://pc-index.org.

Thus, although the IMF $B_{y}$-related variations in the polar cap magnetic fields maximize at noon, the $\triangle \mathrm{PCN}_{\mathrm{SS}}$ values at this local time are quite small due to the projection to the optimum direction in combination with the high slope values. The real IMF $B_{y}$-related variations in the polar cap magnetic fields are almost absent during the night and morning hours as seen clearly in Fig. 3c as well as in the statistical values in Fig. 2. Hence, it is not appropriate that the unjustified solar wind sector-related $\triangle \mathrm{PCN}_{\mathrm{SS}}$ values are so large in the night and morning hours, here almost $2.5 \mathrm{mV} \mathrm{m}^{-1}$ in the index values through 03:00-09:00 UT, for a case with IMF $B_{y} \sim 4 \mathrm{nT}$, which is a moderate and common value. 


\subsection{Comparisons PCN-IAGA and Em-OMNI data}

For comparison of specific data sets, OMNI solar wind data referred to the magnetospheric nose have been downloaded from the omniweb site (ftp://omniweb.gsfc.nasa.gov/omni/) while PCN index values derived according to the new IAGAadopted PC index procedure have been downloaded from the http://pc-index.org (2014) website.

In order to get a clear view of the effects of the solar wind sector terms, values of the northern Polar Cap (PCN) index and the geo-effective electric field $\left(E_{\mathrm{M}}\right)$ shifted in time to apply to the polar cap have been contrasted in plots of which some examples are shown here. Both quantities have fluctuations. Hence, for a fair comparison some averaging is needed.

From the diagram in Fig. 6b of JT2011 a period of positive excursion in the $\Delta H_{\mathrm{SS}}$ term could be the interval from 18 to 25 June 2001 with peak value on 22 June, the date used for Fig. 4 here. For this interval the series of $E_{\mathrm{M}}$ as well as PCN values have been averaged over corresponding times through the 8 days. The 8-day mean values are displayed in Fig. 5a. From Fig. 6b of JT2011 a corresponding interval for negative excursions in $\Delta H_{\mathrm{SS}}$ could be from 3 to 10 June, 2001. The 8-day average values for this interval are presented in Fig. 5b.

In Fig. 5a it is seen that between 03:00 and 09:00 UT (local night at Thule) the PCN index values are much lower, by around 1.0 to $1.5 \mathrm{mV} \mathrm{m}^{-1}$, than the corresponding $E_{\mathrm{M}}$ values (in $\mathrm{mV} \mathrm{m}^{-1}$ ). This agrees well with the values of the anticipated depression, $\triangle \mathrm{PCN}_{\mathrm{SS}}$, seen within this time interval in Fig. 4 bearing in mind that the values on 22 June represent the peak excursion with $\Delta H_{\mathrm{SS}} \sim 65 \mathrm{nT}$ in Fig. $6 \mathrm{~b}$ of JT2011. The $\Delta D_{\mathrm{SS}}$ values also needed for calculation of PCN index values vary similarly to $\Delta H_{\mathrm{SS}}$ as seen in Fig. 4 of JT2011.

For the night hours of the second interval, 3-10 June 2001, the data displayed in Fig. $5 \mathrm{~b}$ indicate the opposite trend compared to Fig. 5a for the relation between PCN and $E_{\mathrm{M}}$. Now, the average $\mathrm{PCN}$ values are larger than the corresponding $E_{\mathrm{M}}$ values by 0.5 to $1.0 \mathrm{mV} \mathrm{m}^{-1}$. Considering that the $\Delta H_{\mathrm{SS}}$ values range from $\sim-20$ to $-35 \mathrm{nT}$ during this interval and that the $\Delta D_{\mathrm{SS}}$ values are also lower than in the foregoing interval there is again agreement with the expectations provided by Fig. 4.

There are also some systematic differences in the relations between PCN and $E_{\mathrm{M}}$ during daytime in the two cases (cf. Fig. 12 in Sect. 7.3 on reverse convection). The important point here is the documentation that during night hours the handling of the solar wind sector terms ( $\Delta H_{\mathrm{SS}}$ and $\left.\Delta D_{\mathrm{SS}}\right)$ used in the "effective" QDC derivation in new IAGAendorsed procedure has adverse consequences. The solar wind sector terms, which give small contributions at the dayside, may give quite substantial, but unjustified changes in the PC index values when used at the night side.
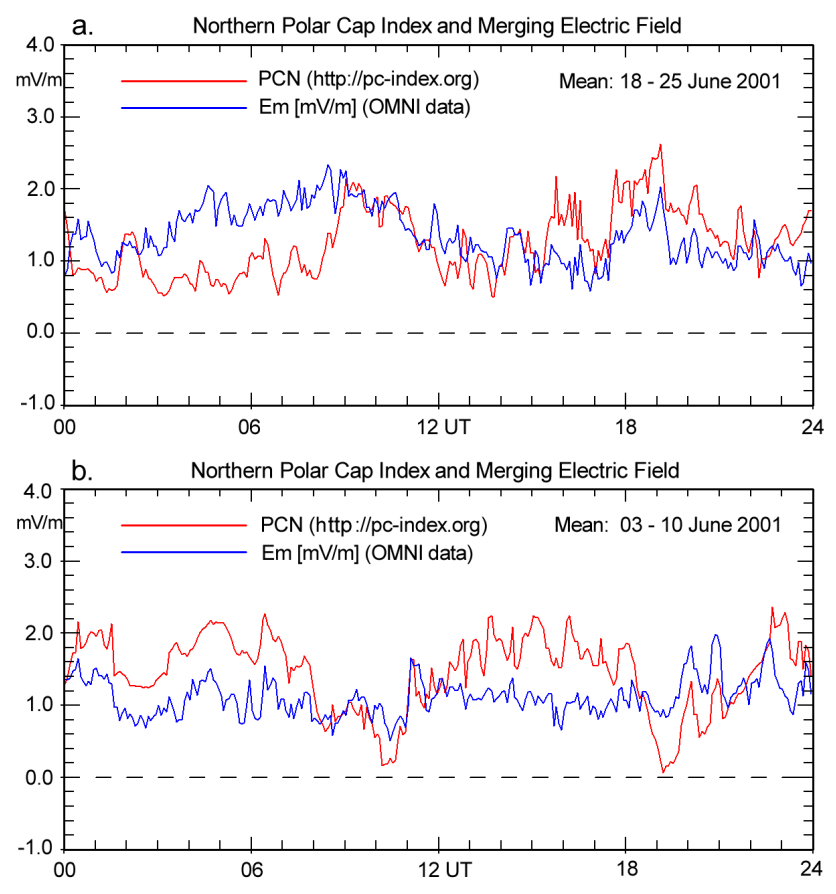

Figure 5. Display of 8-day averages of PCN-IAGA indices and $E_{\mathrm{M}^{-}}$ OMNI values. (a) 18-25 June 2001 for positive $\Delta H_{\text {SS }}$. (b) 3-10 June 2001 for negative $\Delta H_{\mathrm{SS}}$.

\section{Alternative QDC procedure}

A different QDC derivation procedure built from the principles described in TJS2006 and JT2008 is outlined in Stauning (2011). From TJS2006 the principle of using the quietest data values, like those quoted in Sect. 2.3, is implemented. From JT2008 the variability and gradients in the data are considered to be useful parameters for the selection of quiet samples. In addition, the quiet samples are weighted to give preference to cases where the same face of the sun is in view.

The QDC- $H$ values derived by this "Solar Rotation Weighted" (SRW) procedure (Stauning, 2011) are displayed by the red line in Fig. 6 superposed on the $H$-component values in blue line corresponding to the signature used in Fig. 4.10 of TJ2012 (Fig. 1 of JT2008). At the bottom of Fig. 6 the smoothed IMF $B_{y}$ values are displayed in magenta line relating to the scale shown to the right.

The range in dates from day 145 to 245 is the same as in Fig. 1 of JT2011 (or Fig. 4.10 of TJ2012). Hence the IMF $B_{y}$ values displayed at the bottom of Fig. 6 could also be used for judging the IMF $B_{y}$-related QDC- $H$ excursions. Comparing the QDC values in Fig. 1 of JT2011 (or Fig. 4.10 of TJ2012) and Fig. 6, note the two proper features of the QDCs presented in Fig. 6 (in contrast to the corresponding features in Fig. 1 of JT2011 (or Fig. 4.10 of TJ2012):

A. The rather constant top levels of the QDC- $H$ traces in Fig. 6 represent night values of the quiet magnetic field 


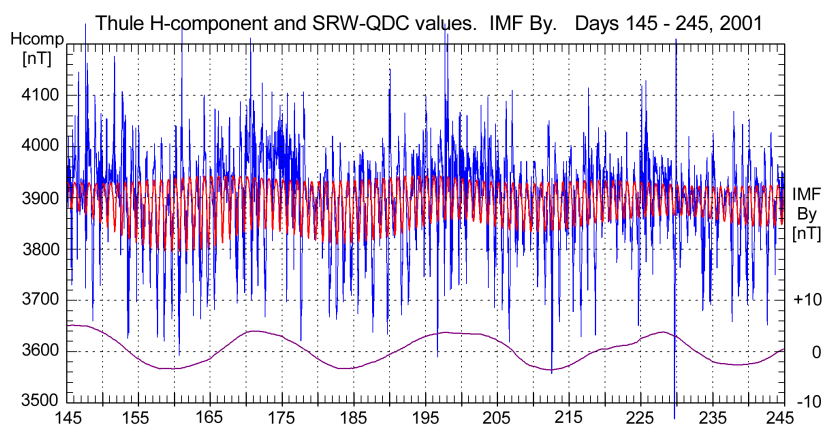

Figure 6. Thule $H$-component in blue line with the "solar rotation weighted" (SRW) QDC- $H$ component superposed in red. (derived by the procedure described in Stauning, 2011).

not significantly affected by the IMF $B_{y}$-variations (cf. Figs. 2 and 3c).

B. The amplitudes in the daily range of the QDC- $H$ traces seen in Fig. 6 are quite strongly affected by the IMF $B_{y}$ variations since the real daily variation in the magnetic field are significantly different for positive and negative IMF $B_{y}$ cases (see Figs. 2 and 3).

This QDC procedure may not be perfect but it provides considerable improvements compared to the IAGA-endorsed "effective" QDC procedure, particularly for the night-time QDC levels.

\section{The reverse convection problem for $\mathrm{PC}$ index coefficients and index values}

During cases of strong northward interplanetary magnetic fields (IMF $B_{z}>>0$ ) the transpolar ionospheric convection (DP3 mode, cf. Fig. 1) and the associated magnetic variations are often opposite (reverse) of the usual forward (DP2) convection and could be quite strong. The proportionality between the magnetic variations projected to the optimum direction and the geo-effective (or "merging") solar wind electric field, which is assumed in the definition of a polar cap index (cf. Eq. 3), breaks down. The (negative) projected magnetic variations could be quite large, numerically, while the geo-effective electric field (cf. Eq. 2) approaches zero for purely northward IMF regardless of its magnitude.

\subsection{Forward and reverse convection intensities at Thule and Vostok}

Figure 7 presents the statistics for the occurrence frequencies and strengths of strong reverse convection cases through the data interval from 1997 to 2009 used in the recent IAGAendorsed PC index procedure. Here, the strong reverse convection cases are defined as those where the (negative) value of the horizontal magnetic term projected to the optimum direction, $F_{\mathrm{PROJ}}$, is more than $50 \mathrm{nT}$ below the projected quiet

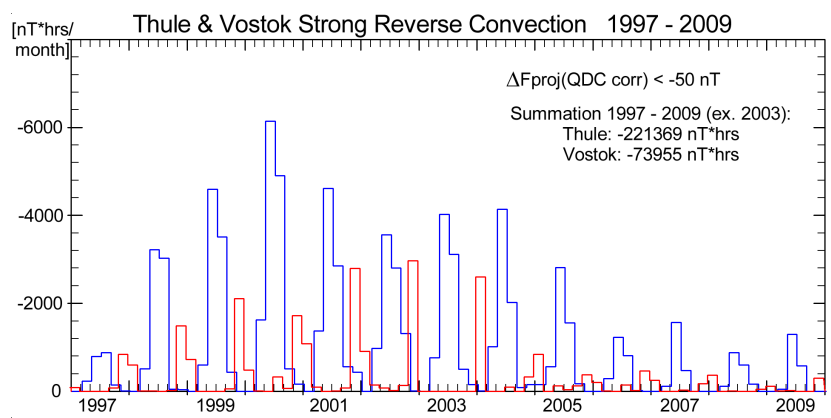

Figure 7. Strong reverse convection cases illustrated by the monthly sums of intensity times duration $([\mathrm{nT} \times \mathrm{h}])$ for strongly negative values $\left(\Delta F_{\mathrm{PROJ}}(\mathrm{QDC}\right.$ corr $\left.)<-50 \mathrm{nT}\right)$ of the projected horizontal deviations for Thule (blue line) and Vostok (red). (Note: no Vostok data in 2003).

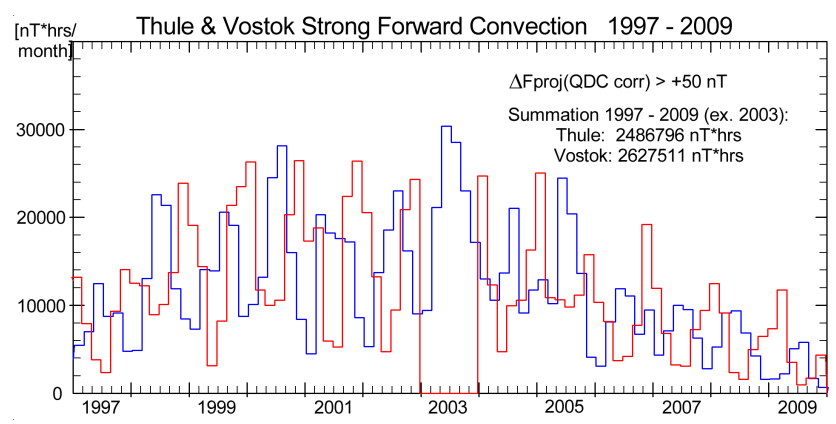

Figure 8. Forward convection cases illustrated by the monthly sums of intensity times duration $[\mathrm{nT} \times \mathrm{h}]$ ) for strongly positive values $\left(\Delta F_{\mathrm{PROJ}}(\mathrm{QDCcorr})>+50 \mathrm{nT}\right)$ of the projected horizontal variation for Thule (blue line) and Vostok (red). (Note: no Vostok data in 2003).

level. The figure presents the monthly sums (smoothed over 2 months) of the product of the QDC-corrected and projected field values and their duration $[\mathrm{nT} \times \mathrm{h}]$ for Thule (blue line) and Vostok (red line).

For Thule and for Vostok the occurrence frequency of reverse convection cases peaks in the local summer months. Furthermore, from Fig. 7 it is clear that the occurrence frequency and intensity of reverse convection cases are much larger at Thule compared to Vostok. The summations over the entire span of years (excluding year 2003, no data from Vostok) give 3 times the intensity $\times$ duration value at Thule $(-221369 \mathrm{nT} \times \mathrm{h})$ compared to Vostok $(-73955 \mathrm{nT} \times \mathrm{h})$.

For comparison, Fig. 8 presents the corresponding display for strong forward convection cases $\left(F_{\mathrm{PROJ}}\right.$ more than $50 \mathrm{nT}$ above the projected quiet level) and for the same span of years (1997-2009). From Fig. 8 it is seen that the intensities of forward convection cases are about the same for Thule and Vostok. The summations over the entire span of years (excluding year 2003) give an intensity $\times$ duration value at Thule of $2486796 \mathrm{nT} \times \mathrm{h}$ slightly less than that for Vostok of $2627511 \mathrm{nT} \times \mathrm{h}$. 

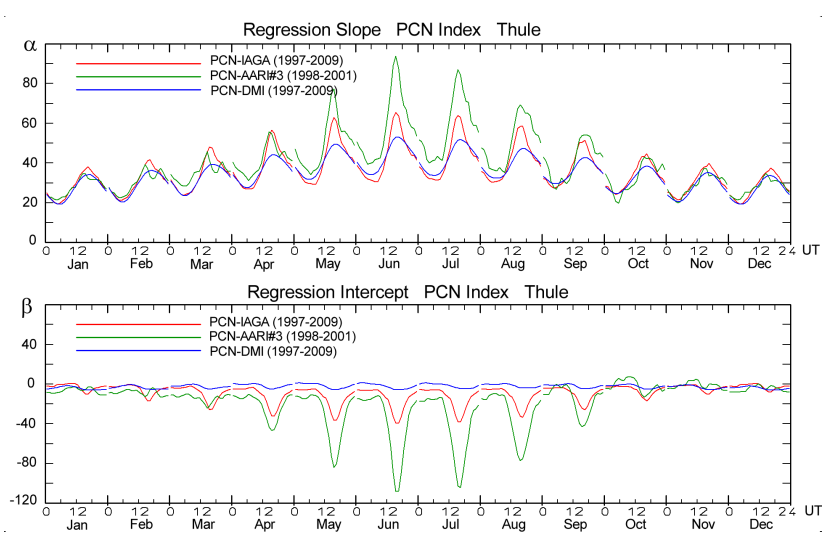

Figure 9. Slopes (upper field) and intercepts (bottom field) from PCN derivation provided in http://pc-index.org (IAGA endorsed) (red lines), derived by Troshichev et al. (2006) (green lines), and derived by DMI (blue lines). Each field has 12 columns to display monthly means of the variations 00:00 to 24:00 UT.

\subsection{Comparison of $\mathrm{PCN}$ and PCS regression coefficients}

Including reverse convection cases adds to the "noise level" in the calculations of the optimum angle determined by the bulk of forward convection cases but the changes in angles are small. For the regression coefficients, however, including reverse convection events gives substantial increases in the slope values and negative increases in the intercept values. The effects from reverse convection events on the regression are illustrated in Fig. 11a and b of Stauning (2013b). The effects are particularly strong at daytime in the summer season and much larger for Thule than for Vostok due to the larger frequency and strength of reverse convection events in the northern polar cap compared to the southern. In addition to the direct consequences, in particular for the PCN index values, including the reverse convection cases causes strong imbalance between PCN and PCS coefficients and values.

The effects of including reverse convection cases in the data base for the regression calculations are illustrated in Fig. 9. In each of the fields for slopes (upper field) and intercepts (bottom field) the diagram holds a section for each of the 12 months of a year. The curves within 1 monthly section define the coefficient values through the $24 \mathrm{~h}$ of a day averaged over the month in question. They are shown in different line colours for the three versions. The IAGA-endorsed version (from http://pc-index.org) in red line is based on data from 1997 to 2009. The AARI\#3 version in green line is based on data from 1998 to 2001 (TJS2006). Both versions include reverse convection cases. The DMI version in blue line is based on data from 1997 to 2009 excluding strong reverse convection events. A similar figure without the IAGAendorsed parameters and using a different epoch for the DMI coefficients is provided in Stauning (2013b).
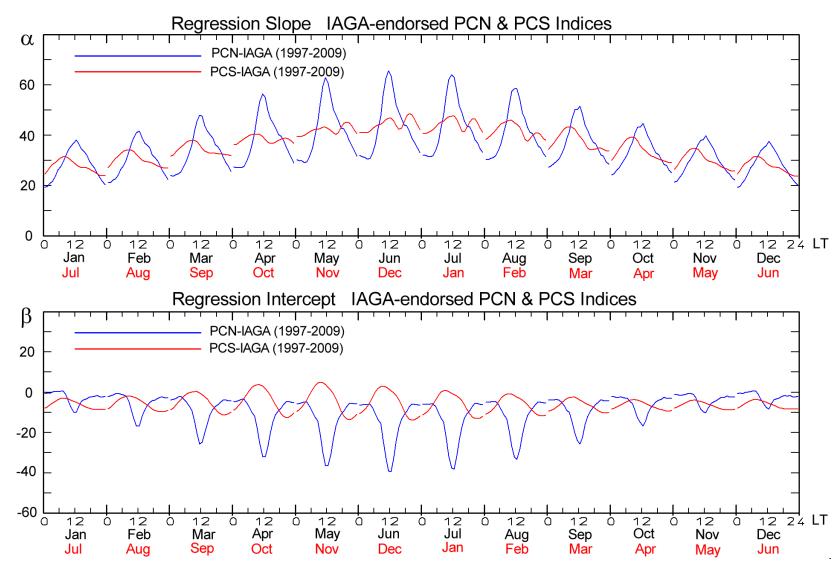

Figure 10. Slope and regression coefficients for $\mathrm{PCN}$ (blue) and PCS (red line) from the IAGA-endorsed PC index procedure. Data from Thule, Vostok and OMNI 1997-2009. Reverse convection cases included (Index coefficients from http://pc-index.org, 2014).
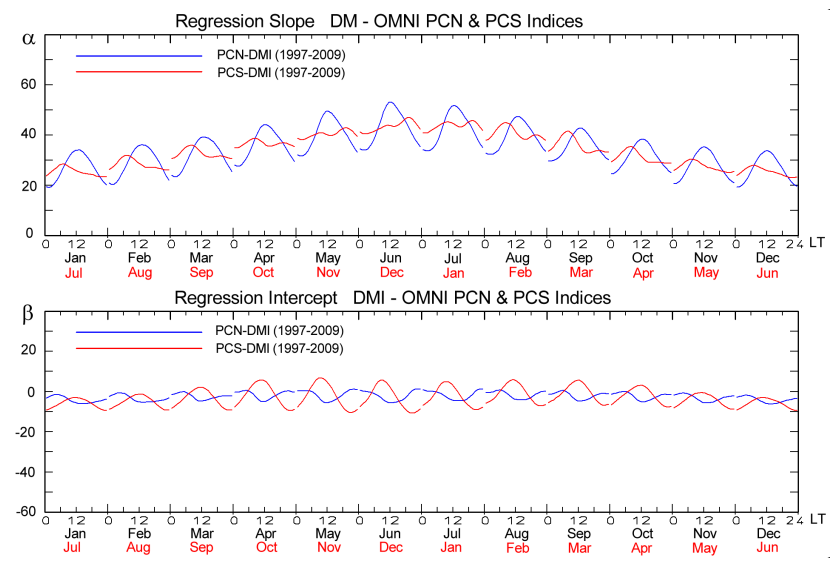

Figure 11. Slope and regression coefficients for $\mathrm{PCN}$ (blue) and PCS (red line) derived with DMI procedure. Data from Thule, Vostok, and OMNI 1997-2009 (ex. 2003). Strong reverse convection cases excluded.

The extended data base interval for the IAGA version compared to the AARI\#3 version now includes the less active years 1997 and 2002-2009. Referring to Fig. 7 it is easy to see that the abundance of reverse convection cases is much smaller in these years than in the very active years from 1998 to 2001. Hence, the relative importance of reverse convection cases is reduced from the AARI\#3 (green lines) to the IAGA version (red lines) in Fig. 9, and the consequences are reduced slopes and less negative intercept values particularly at midday in the summer months. These changes are in strong contrast to the general conclusion in Troshichev et al. (2011a) on the invariability of PC index coefficients regardless of epoch. The PCS coefficients may remain about the same but the PCN coefficients change substantially with changing data epoch. 
For illustration of the inter-hemispherical balance, Fig. 10 presents the PCN and PCS regression parameters, slopes and intercepts, derived from the present IAGA-endorsed coefficient files (http://pc-index.org). For easy comparison, the regression parameters have now been plotted vs. local solar time by advancing the PCN coefficients by $4 \mathrm{~h}$ and delaying the PCS coefficients by $7 \mathrm{~h}$ corresponding to the different longitudes of the two stations. Furthermore, the seasonal axis for the PCS index (in red) has been shifted by 6 months to make the seasonal variations in the PCS traces correspond to the equivalent variations in the PCN traces. It is now easy to see that the correspondence between PCN and PCS coefficients is not good.

Figure 11 presents the corresponding diagrams for the PCN and PCS coefficients derived by the DMI procedure omitting strong reverse convection events, but using the same span of years (1997-2009, ex. 2003), the same geomagnetic data from Thule and Vostok, respectively, and the same OMNI data as those used in the IAGA-endorsed procedure. The traces are again plotted vs. local time and season like in Fig. 10. A similar diagram based on data from the epoch 1995-2005 was published in Stauning (2013b).

From a comparison between the plots in Figs. 10 and 11, two features emerge. Firstly, the DMI procedure provides much better agreement between PCN and PCS index coefficients than the IAGA-endorsed procedure. Secondly, the sets of PCS coefficients agree fairly well between the IAGAendorsed version and the DMI version, whereas there are large differences between the two sets of slope and intercept coefficients for the PCN index. The main reason for this discrepancy is the inclusion of strong reverse convection cases in the IAGA-endorsed procedure in combination with the much larger frequency and intensity of strong reverse convection events at Thule compared to Vostok.

Table 2 provides a summary of the effects from strong reverse convection on the peak values of the slope and intercept coefficients. For the AARI\#3 version (epoch 1998-2001) and the IAGA-endorsed (http://pc-index.org) version (epoch 1997-2009) the ratios of strong reverse to strong forward convection intensities have been calculated from events with projected deviation from QDC in the horizontal component exceeding $100 \mathrm{nT}$. The events were extracted within local summer months (June-July for Thule, December-January for Vostok) and within local midday hours (12:00-20:00 UT for Thule, 04:00-12:00 UT for Vostok). For the DMI version for epoch 1997-2009, the strong reverse convection cases have been screened away in the regression procedure.

Note the strong decrease in the peak slope parameter with the decreasing relative amount of reverse convection events and the corresponding decrease in the numerical value of the (negative) intercept parameter. Also note the improved match between slope and intercept parameters for the northern (PCN) and southern (PCS) indices obtained by omitting the strong reverse convection cases in the DMI version.

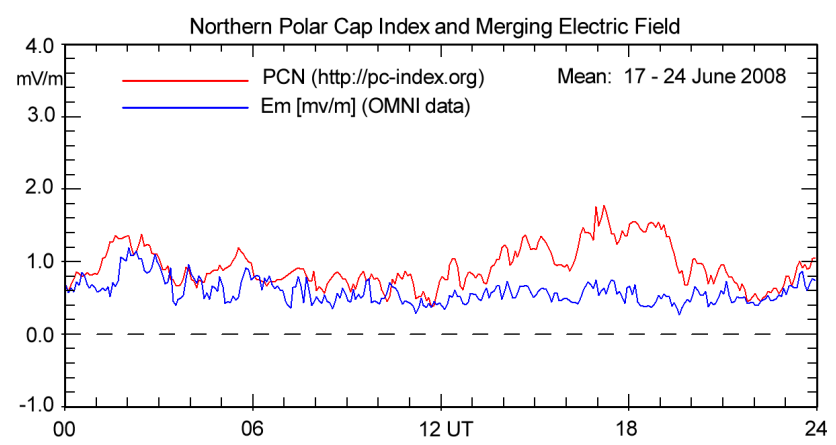

Figure 12. Display of 8-day averages of PCN-IAGA indices (red line) and $E_{\mathrm{M}}$-OMNI values (blue line) for the quiet interval 17-24 June 2008.

Referring to the defining equation (Eq. 3) for the PC index, a larger slope gives reduced PC index values for the strong events where the intercept contribution is relatively small compared to the disturbance. Conversely, for weak events, where the projected disturbance, $\Delta F_{\mathrm{PROJ}}$, is small, an increased negative intercept value gives larger PC index values. With the present IAGA-endorsed regression coefficients, the large intercept values for Thule give PCN index values that on the average are around $0.5-1.0 \mathrm{mV} \mathrm{m}^{-1}$ too large compared to the related geoeffective electric field values during quiet summer daytime conditions.

\subsection{Example case of unjustified PC index enhancement during quiet conditions}

An example of unjustified PC index enhancements for a quiet interval (17-24 June 2008) is presented in Fig. 12. The blue line indicates the (weak) average geoeffective electric field through $24 \mathrm{~h}$ determined from OMNI data while the red line presents the mean IAGA-endorsed PCN values taken from http://pc-index.org. The two quantities should, on the average, be equal according to the definition of the PC index. During midday hours (12:00-22:00 UT) the average PCN index values are up to $1 \mathrm{mV} \mathrm{m}^{-1}$ larger than the corresponding electric field values. The increased PCN level is not justified in the magnetic variations, which are very small at this time, but just the result of the large negative intercept regression parameter that was derived with the inclusion of strong reverse convection cases.

At the date and time at the middle of the interval of enhanced index values (20 June, 16:00 UT) the IAGA-endorsed coefficients according to http://pc-index.org are $\alpha=65.61$, $\beta=-40.89$ (cf. Fig. 10). Hence, the addition to the PC index coming just from the coefficients, notably the large (negative) intercept value, is $\Delta \mathrm{PC}=-\beta / \alpha=0.62 \mathrm{mV} \mathrm{m}^{-1}$ which is in agreement with the display in Fig. 12. 
Table 2. Ratio of strong reverse to forward convection intensities and extreme values of slope $(\alpha)$ and intercept $(\beta)$ coefficients for various index versions and epochs.

\begin{tabular}{lllrcl}
\hline Version & Index & Epoch & ${ }^{\mathrm{a}} \mathrm{Rev} / \mathrm{Fwd}$ & $\alpha\left[\mathrm{nT} / \mathrm{mV} \mathrm{m}^{-1}\right]$ & $\beta[\mathrm{nT}]$ \\
\hline AARI\#3 (2006) & PCN & $1998-2001$ & $40.1 \%$ & 96.4 & -117.0 \\
pc-index.org & PCN & $1997-2009$ & $31.0 \%$ & 66.0 & -41.0 \\
pc-index.org & PCS & $1997-2009$ & $4.7 \%$ & 48.0 & -15.0 \\
Dmi (2015) & PCN & $1997-2009$ & $\mathrm{~b}_{0} \%$ & 53.2 & -5.6 \\
Dmi (2015) & PCS & $1997-2009$ & $\mathrm{~b}_{0} \%$ & 47.2 & -10.7 \\
\hline
\end{tabular}

${ }^{\mathrm{a}}$ Forward and reverse convection intensities are calculated for Thule: June-July, 12:00-20:00 UT; and for Vostok: December-January, 04:00-12:00 UT. Projected deviations > 100 nT from QDC.

${ }^{\mathrm{b}}$ For these two versions the strong reverse convection events are screened away in the regression.

\section{Discussions}

It was shown (cf. Sect. 5.2) that the IMF $B_{y}$-related solar sector (SS) term added to the basic QDC can produce up to $2.4 \mathrm{mV} \mathrm{m}^{-1}$ unjustified changes in the PC index values (cf. Fig. 4 and Table 1) during night and morning hours, where the real IMF $B_{y}$ effects are negligible (cf. Figs. 2 and 3c). Furthermore, the pre-processing step where the SS term is subtracted from the component data used in the basic QDC procedure (cf. Sect. 3.2) is problematic. If the quiet samples are clustered in either the positive or the negative phase of the IMF $B_{y}$ variations over the 30-day interval then the SS effect on the basic QDC may further reduce or enhance the resulting SS contribution to the $\mathrm{PC}$ indices.

To judge the importance of SS-related index modifications, note from Troshichev et al. (2011b): "It has been found that all examined storms, lying in (Dst) range from -30 to $-373 \mathrm{nT}$, started when the PC index and, correspondingly, the $E_{\mathrm{M}}$ field firmly exceeded the threshold $>2 \mathrm{mV} \mathrm{m}^{-1}$." Thus, unjustified changes in the PC index of magnitude up to $2.4 \mathrm{mV} \mathrm{m}^{-1}$ (or possibly more), which could go both ways, might hide the start of geomagnetic storms or, conversely, could indicate storm development during completely calm conditions. And there is no guarantee that the presented examples have found the most extreme cases.

Concerning the NBZ-related reverse convection issue, the PC indices are meant to scale the forward transpolar (DP2) convection in response to the solar wind forcing by using the projection of magnetic variations to the forward convection-dominated "optimum direction" (cf. Troshichev et al., 1988, 2006). The large negative values of the projected variations during strong reverse convection events associated with northward IMF conditions disturb the least squares regression of magnetic variations against the non-negative solar wind merging electric field in the derivation of index coefficients.

It is documented here that strong reverse convection events are much more frequent at Thule (PCN index) than at Vostok (PCS index) (cf. Fig. 7). Furthermore, it is shown how the PCN index coefficients respond to a reduction in the relative abundance of reverse convection events going from so- lar maximum epoch 1998-2001 (Troshichev et al., 2006) to epoch 1997-2009 (http://pc-index.org), and in a further step omitting the strong reverse convection events (cf. Fig. 9). The peak slope (at noon in summer) is reduced from 96 through 66 to $53 \mathrm{nT} / \mathrm{mV} \mathrm{m}^{-1}$ while the intercept is reduced from -117 through -41 to $-6 \mathrm{nT}$ (cf. Table 2 ). The latter set of parameters is in concordance with the corresponding parameters for the PCS index.

The effects from such major coefficient changes could be assessed from the formula provided in Eq. (3). With the new IAGA-endorsed coefficients for Thule, the calculated PCN index values could be reduced by up to $24 \%$ during disturbed conditions due to the inclusion of strong reverse convection events in the regression procedure, while index values during calm conditions may be given unjustified values of 0.5 to $1 \mathrm{mV} \mathrm{m}^{-1}$ during midday hours in the summer months (cf. Table 2 and Fig. 12). With the IAGA-endorsed coefficients for Vostok the "quiet" PCS values are close to zero throughout the day and year.

To judge the importance of such systematic coefficientbased index changes, for instance, in conjugate studies, note that the yearly average of positive PCN values (from http: $/ / \mathrm{pc}$-index.org) ranges from $1.95 \mathrm{mV} \mathrm{m}^{-1}$ during a solar max year (2003) to $0.99 \mathrm{mV} \mathrm{m}^{-1}$ during a solar min year (2008).

\section{Conclusions}

The use of IMF $B_{y}$-related solar wind sector (SS) terms derived from the daily median component values in the IAGAadopted PC index procedure is based on the erroneous concept that the IMF- $B_{y}$ effects are the same through day and night. The adverse consequences are

- uncertain IMF- $B_{y}$ effects in the derivation of basic QDC values

- unjustified positive or negative contributions to the PC indices of up to more than $2 \mathrm{mV} \mathrm{m}^{-1}\left(2.4 \mathrm{mV} \mathrm{m}^{-1}\right.$ in example case).

The inclusion of NBZ-related strong reverse convection cases in the data base used to calculate PC index coefficients 
(slope and intercept) has some adverse consequences particularly for the PCN index:

- the coefficients for PCN depend critically on the amount of solar maximum or solar minimum intervals included in the data base

- reduction by up to $24 \%$ in $\mathrm{PCN}$ index values during strong disturbances

- unjustified contributions of $0.5-1.0 \mathrm{mV} \mathrm{m}^{-1}$ to daytime PCN index values during quiet conditions

- imbalance between PCN and PCS index coefficients and index values.

It is acknowledged that no index is perfect and also that having an internationally agreed PC index is a valuable asset. However, noting that index average values range from 1 to 2 , and that magnetic storms generally start at PC index values exceeding $2 \mathrm{mV} \mathrm{m}^{-1}$, the adverse effects related to the QDC derivation and to the handling of reverse convection events seriously devaluate the present IAGA-endorsed PC indices and make them less useful for space weather monitoring as well as for scientific analyses of solar wind-magnetosphere interactions.

Previously published alternative procedures for handling of the QDC and reverse convection problems to avoid the adverse effects are outlined here.

\section{Data availability}

Live PC index values and PCN and PCS index series are now made available through the new web site: http://pc-index.org. Furthermore, it holds PCN and PCS index coefficients derived by the IAGA-endorsed procedure. QDC values are not included. The web site includes the document "Polar Cap (PC) Index" written by O. A. Troshichev.

The Space-DTU (2014) ftp web site for PC indices: ftp://ftp.space.dtu.dk/WDC/indices/pcn/PC_index_ IAGA_endorsement_documentation/ includes the documents: PC_index_description_main_document.pdf and PC_index_description_Appendix_A.pdf, and a directory, PC_index_description_Appendix_A_file_archive, with program transcripts and data files (neither including QDC values nor solar wind sector terms).

Acknowledgements. The observatories in Qaanaaq and Vostok and their supporting institutes are gratefully acknowledged for providing high-quality geomagnetic data for this study.

The topical editor G. Balasis thanks three anonymous referees for help in evaluating this paper.

\section{References}

Chun, F. K., Knipp, D. J., McHarg, M. G., Lu, G., Emery, B. A., Vennerstrøm, S., and Troshichev, O. A.: Polar cap index as a proxy for hemispheric Joule heating, Geophys. Res. Lett., 26 , 1101-1104, doi:10.1029/1999GL900196, 1999.

Chun, F. K., Knipp, D. J., McHarg, M. G., Lacey, J. R., Lu, G., and Emery, B. A.: Joule heating patterns as a function of polar cap index, J. Geophys. Res., 107, SIA 8-1-SIA 8-9, doi:10.1029/2001JA000246, 2002.

Fiori, R. A. D., Koustov, A. V., Boteler, D., and Makarevich R. A.: PCN magnetic index and average convection velocity in the polar cap inferred from SuperDARN radar measurements, J. Geophys. Res., 114, A07225, doi:10.1029/2008JA013964, 2009.

Gao, Y., Kivelson, M. G., Ridley, A. J., Weygand, J. M., and Walker, R. J.: Utilizing the polar cap index to explore strong driving of polar cap dynamics, J. Geophys. Res., 117, A07213, doi:10.1029/2011JA017087, 2012.

Huang, C.-S.: Variations of polar cap index in response to solar wind changes and magnetospheric substorms, J. Geophys. Res., 110, A01203, doi:10.1029/2004JA010616, 2005.

Janzhura, A. S. and Troshichev, O. A.: Determination of the running quiet daily geomagnetic variation, J. Atmos. Sol.-Terr. Phy., 70, 962-972, doi:10.1016/j.jastp.2007.11.004, 2008.

Janzhura, A. S. and Troshichev, O. A.: Identification of the IMF sector structure in near-real time by ground magnetic data, Ann. Geophys., 29, 1491-1500, doi:10.5194/angeo-29-14912011, 2011.

Janzhura A., Troshichev, O. A., and Stauning, P.: Unified PC indices: Relation to the isolated magnetic substorms, J. Geophys. Res., 112, A09207, doi:10.1029/2006JA012132, 2007.

Kan, J. R. and Lee, L. C.: Energy coupling function and solar wind-magnetosphere dynamo, Geophys. Res. Lett., 6, 577-580, doi:10.1029/GL006i007p00577, 1979.

Liou, K., Carbary, J. F., Newell, P. T., Meng, C.-I., and Rasmussen, O.: Correlation of auroral power with the polar cap index, J. Geophys. Res., 108, 1108, doi:10.1029/2002JA009556, 2003.

Lukianova, R.: Magnetospheric response to sudden changes in solar wind dynamic pressure inferred from polar cap index, J. Geophys. Res., 108, 1428, doi:10.1029/2002JA009790, 2003.

Ridley A. J. and Kihn, E. A.: Polar cap index comparisons with AMIE cross polar cap potential, electric field, and polar cap area, Geophys. Res. Lett., 31, L07801, doi:10.1029/2003GL019113, 2004.

Stauning, P.: Determination of the quiet daily geomagnetic variations for polar regions, J. Atmos. Sol.-Terr. Phy., 73, 2314-2330, doi:10.1016/j.jastp.2011.07.004, 2011.

Stauning, P.: Comments on quiet daily variation derivation in "Identification of the IMF sector structure in near-real time by ground magnetic data" by Janzhura and Troshichev (2011), Ann. Geophys., 31, 1221-1225, doi:10.5194/angeo-31-1221-2013, 2013a.

Stauning, P.: The Polar Cap index: A critical review of methods and a new approach, J. Geophys. Res.-Space, 118, 5021-5038, doi:10.1002/jgra.50462, 2013b.

Stauning, P.: Power grid disturbances and polar cap index during geomagnetic storms, J. Space Weather Space Clim. 3, A22, doi:10.1051/swsc/2013044, 2013c.

Stauning, P., Troshichev, O. A., and Janzhura, A.: The Polar Cap (PC) indices. Relations to solar wind parameters and global magnetic activity, J. Atmos. Sol.-Terr. Phy., 70, 18, 2246-2261, doi:10.1016/j.jastp.2008.09.028, 2008. 
Takalo, J. and Timonen, J.: Neural network prediction of the AE index from the PC index, Phys. Chem. Earth. Pt. C, 24, 89-92, doi:10.1016/S1464-1917(98)00013-0, 1999.

Troshichev, O. A. and Andrezen, V. G.: The relationship between interplanetary quantities and magnetic activity in the southern polar cap, Planet. Space Sci., 33, 415-419, 1985.

Troshichev, O. A. and Janzhura, A.: Relationship between the PC and $\mathrm{AL}$ indices during repetitive bay-like magnetic disturbances in the auroral zone. J. Atmos. Sol.-Terr. Phy.., 71, 1340-1352, doi:10.1016/j.jastp.2009.05.017, 2009.

Troshichev, O. A. and Janzhura, A.: Space Weather monitoring by ground-based means, Springer Praxis Books, Springer-Verlag Berlin Heidelberg, Germany, 287 pp., doi:10.1007/978-3-64216803-1, 2012.

Troshichev, O. A., Andrezen, V. G., Vennerstrøm, S., and FriisChristensen, E.: Magnetic activity in the polar cap - A new index, Planet. Space Sci., 36, 1095-1102, 1988.

Troshichev, O. A., Hayakawa, H., Matsuoka, A., Mukai, T., and Tsuruda, K.: Cross polar cap diameter and voltage as a function of PC index and interplanetary quantities, J. Geophys. Res., 101, 13429-13435, doi:10.1029/95JA03672, 1996.

Troshichev, O. A., Lukianova, R. Y., Papitashvili, V. O., Rich, F. J., and Rasmussen, O.: Polar Cap index (PC) as a proxy for ionospheric electric field in the near-pole region, Geophys. Res. Lett., 27, 3809-3812, doi:10.1029/2000GL003756, 2000.
Troshichev, O. A., Janzhura, A., and Stauning, P.: Unified PCN and PCS indices: method of calculation, physical sense and dependence on the IMF azimuthal and northward components, J. Geophys. Res., 111, A05208, doi:10.1029/2005JA011402, 2006.

Troshichev, O. A., Podorozhkina, N. A., and Janzhura, A. S.: Invariability of relationship between the polar cap magnetic activity and geoeffective interplanetary electric field, Ann. Geophys. 29, 1479-1489, doi:10.5194/angeo-29-1479-2011, 2011a.

Troshichev, O. A., Somarkov, D., and Janzhura, A.: Relation of PC index to the geomagnetic storm Dst variation, J. Atmos. Sol.Terr. Phy.., 73, 611-622, doi:10.1016/j.jastp.2010.12.015, $2011 \mathrm{~b}$.

Troshichev, O., Sormakov, D., and Janzhura, A.: Sawtooth substorms generated under conditions of the steadily high solar wind energy input into the magnetosphere: Relationship between PC, AL and ASYM indices, Adv. Space Res., 49, 872-882, doi:10.1016/j.asr.2011.12.011, 2012.

Vassiliadis, D., Angelopoulos, V., Baker, D. N., and Klimas, A. J.: The relation between the northern polar cap and auroral electrojet geomagnetic indices in the wintertime, Geophys. Res. Lett., 23, 2781-2784, doi:10.1029/96GL02575, 1996.

Vennerstrøm, S., Friis-Christensen, E., Troshichev, O. A., and Andrezen, V. G.: Comparison between the polar cap index PC and the auroral electrojet indices AE, AL and AU, J. Geophys. Res., 96, 101-113, doi:10.1029/90JA01975, 1991. 